Science, Technology and Development 34 (1): 49-51, 2015

ISSN 0254-6418 / DOI: 10.3923/std.2015.49.51

(C) 2015 Pakistan Council for Science and Technology

\title{
Role Played by AKARI-II by Exploring the Lives of Stars in the Space Using Infrared Technology
}

\author{
M. Hanif, M.K. Khattak and M. Aamir \\ Department of Agricultural Mechanization, FCPS, The University of Agriculture Peshawar, Pakistan
}

\begin{abstract}
The study corresponds the role played by AKARI-II for exploring the lives of stars in the space. AKARI-II officially famous as ASTRO-F, launched on 21 February, 2006 is the second Japanese satellite on a space mission to carry out sky-high observations for exploring the lives of stars in the space using infrared technology. It has a telescope fitted with an Infrared camera which has a spectral range of 2-26 $\mu \mathrm{m}$ and the Far-Infrared Surveyor has a range of 50-180 $\mu \mathrm{m}$. The mirror of the telescope is made up of sandwich type silicon carbide, which is kept cool by $180 \mathrm{~L}$ of liquid Helium for sensitive observations. AKARI performs All-sky survey and collect images of very small portions of galaxies in the space. It gives pictures which show the lives of stars. The brighter the stars, the younger are they considered to be.
\end{abstract}

Key words: AKARI-II, sky survey, infrared, telescope

\section{INTRODUCTION}

All the heavenly structures in the universe are widely assumed to be the result of gravitational instability. Galaxies are formed in the dark matter halo and grew under the influence of gravity. The indentation of the ancient circulation of inhomogeneities, although distorted, should be still preserved in the distribution of nowadays galaxies (Matsuura et al., 2011). Therefore, examination of galaxy clustering is crucial to an understanding of the evolution of the primary dark matter field. To make censes of all possibilities of different types of galaxies the infrared technology was introduced and used for exploring galaxies in 1984 (Doi et al., 2009). The infrared emission of light from stars in clusters of galaxies is considered to be a good tracer of star-formation activity. The infrared technology besides a tracer gives a good relationship between the dark matter density and star-formation activity at different epochs. It also gives very good relationship of stars' lives (Onaka et al., 2010). After realizing that infrared imaging is the best way for exploring lives of stars in galaxies AKARI (ASTRO-F) opened novel possibilities to discover the whole sky in the far infrared. AKARI is the second Japanese satellite launched on 21 February, 2006 by the JAXA M-V rocket to provide second generation infrared catalogs to obtain a better spatial resolution and a wider spectral coverage. It is successfully working into a sun-synchronous polar orbit at an altitude of $700 \mathrm{~km}$ and an inclination of $98.20^{\circ}$ (Yamamura and de Jong, 2000).

Telescope of AKARI: The main thing which makes the AKARI a better mission and reliable satellite is its telescope system. AKARI-II uses a Richey-Chretien type telescope, which is $30.6 \mathrm{~kg}$ in weight having an effective diameter of $686 \mathrm{~mm}$ of the primary mirror. The Mirror is made up of sandwich-type silicon carbide Weighing $10.7 \mathrm{~kg}$ (Kawada et al., 2007). The focal length of the mirror is $4197 \mathrm{~mm}$ under an effective collecting area of $287041 \mathrm{~mm}$. The telescope records the data with a high Performance in cold mission having a diffraction-limited at $7.31 \mathrm{~m}$ and in warm mission it is $8.01 \mathrm{~m}$ (Okamura et al., 2014). The AKARI-II satellite and telescope are shown in Fig. $1 \mathrm{a}$ and $\mathrm{b}$.

Focal-plane instruments installed on AKARI: AKARI-II uses two focal plane instruments.

Far-Infrared Surveyor (FIS): The FIS has an Imaging band N60 $\left(65^{1} \mathrm{~m}\right)$, WIDE-S $\left(90^{1} \mathrm{~m}\right)$, WIDE-L $\left(140^{1} \mathrm{~m}\right)$ and N160 $\left(160^{1} \mathrm{~m}\right)$. The Detectors $20 \times 3$ and $20 \times 2 \mathrm{Ge}: \mathrm{Ga}$ arrays for N60 and WIDE-S $15 \times 3$ and $15 \times 2$ stressed Ge: Ga arrays for WIDE-L and N160. The Pixel pitch of 29.5" for N60 and WIDE-S and 49.5" for WIDE-L and N160. It has a Spectroscopy Resolution of $19 \mathrm{~cm}^{-1}$ (Ootsubo et al., 2011).

Infrared Camera (IRC): The IRC uses an Imaging bands N2 $\left(2.4^{1} \mathrm{~m}\right)$, N3 $\left(3.2^{1} \mathrm{~m}\right)$, N4 $\left(4.1^{1} \mathrm{~m}\right) \mathrm{S} 7\left(7^{1} \mathrm{~m}\right)$, S9W $\left(9^{1} \mathrm{~m}\right), \mathrm{S} 11\left(11^{1} \mathrm{~m}\right), \mathrm{L} 15\left(15^{1} \mathrm{~m}\right), \mathrm{L} 18 \mathrm{~W}\left(18^{1} \mathrm{~m}\right)$ and L24 $\left(24^{1} \mathrm{~m}\right)$. The Detectors are $512 \times 412 \mathrm{In} \mathrm{Sb}$ array for NIR 256×256 Si: As arrays for MIR-S/L. The IRC uses a Pixel scale of $1.46 " \times 1.46 "$ for NIR, 2.34"×2.34" for MIR-S and 2.51" $\times 2.39 "$ for MIR-L. All-sky survey is conducted by IRC using S9W and L18W with the effective pixel scale of 10.0" bands under a Spectroscopy Resolution in the range of 0.01-0.17 m (Ootsubo et al., 2011).

Corresponding Author: M. Hanif, Department of Agricultural Mechanization, FCPS, The University of Agriculture Peshawar, Pakistan Tel: +923339049552 

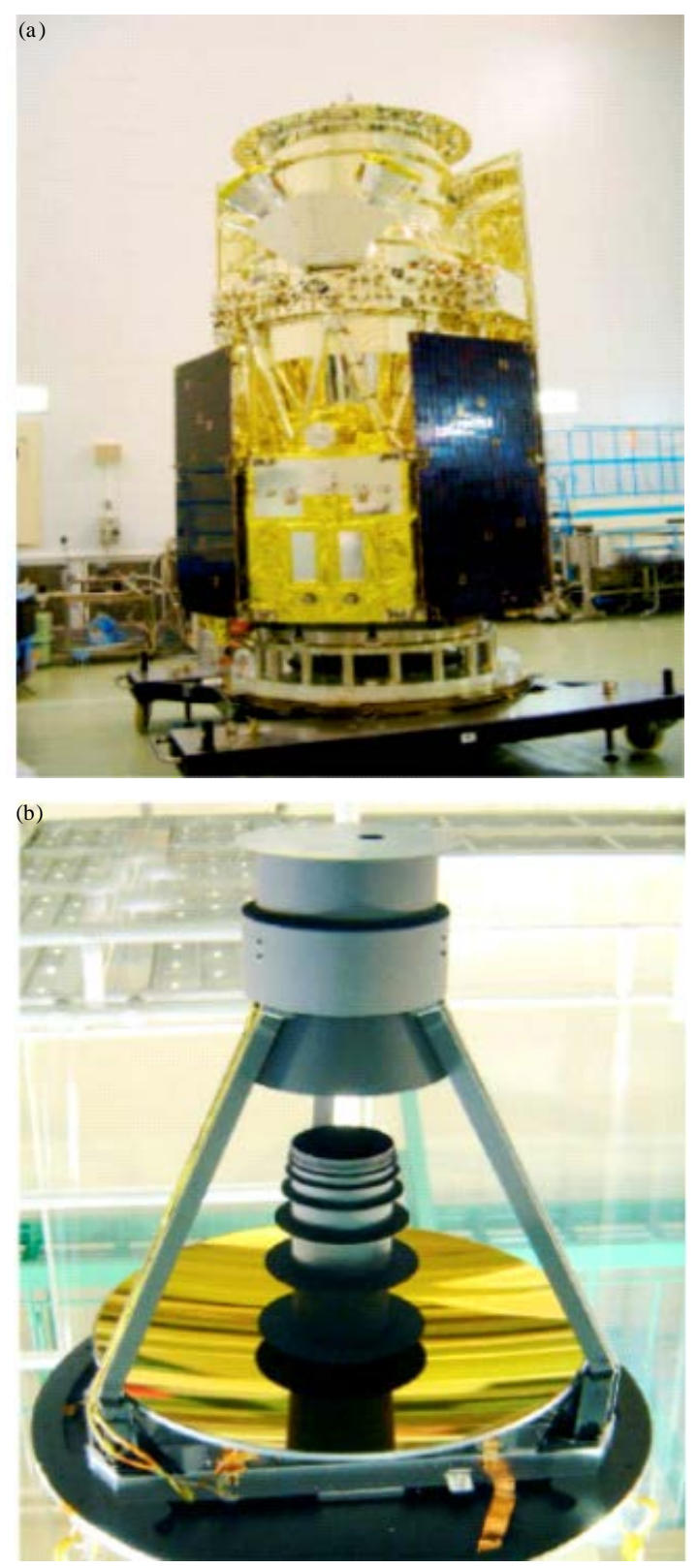

Fig. 1(a-b): AKARI-II (a) Satellite and (b) Telescope

Data recording and reduction by AKARI: AKARI-II starts its data collection from 8th of May, 2006 till 26th of August, 2007 during the life time of cooling medium (Helium). During this period more than $90 \%$ of the entire sky was observed twice at a scan speed of 216 inches $\mathrm{S}^{-1}$. The data calculated at each second was pipeline by a software to make maps. The pointed and reconstructed data was carried out with the data of the Focal Plane Star Sensor of the European Space Astronomy Center (Pollo et al., 2013). AKKARI-II all sky survey photograph is given in Fig. 2 and the dust in the space is given in Fig. 3.

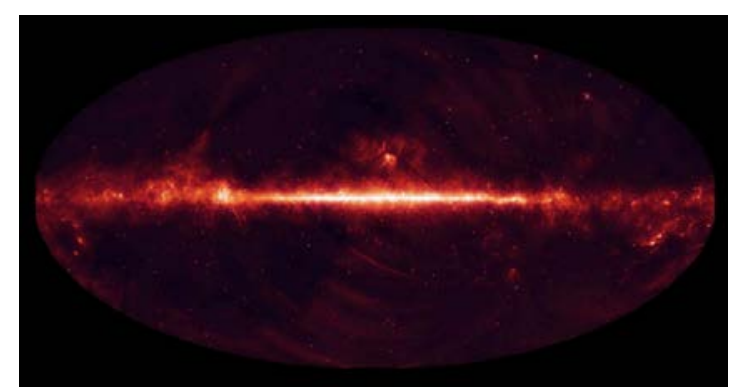

Fig. 2: AKARI-II all sky survey at $9 \mu \mathrm{m}$
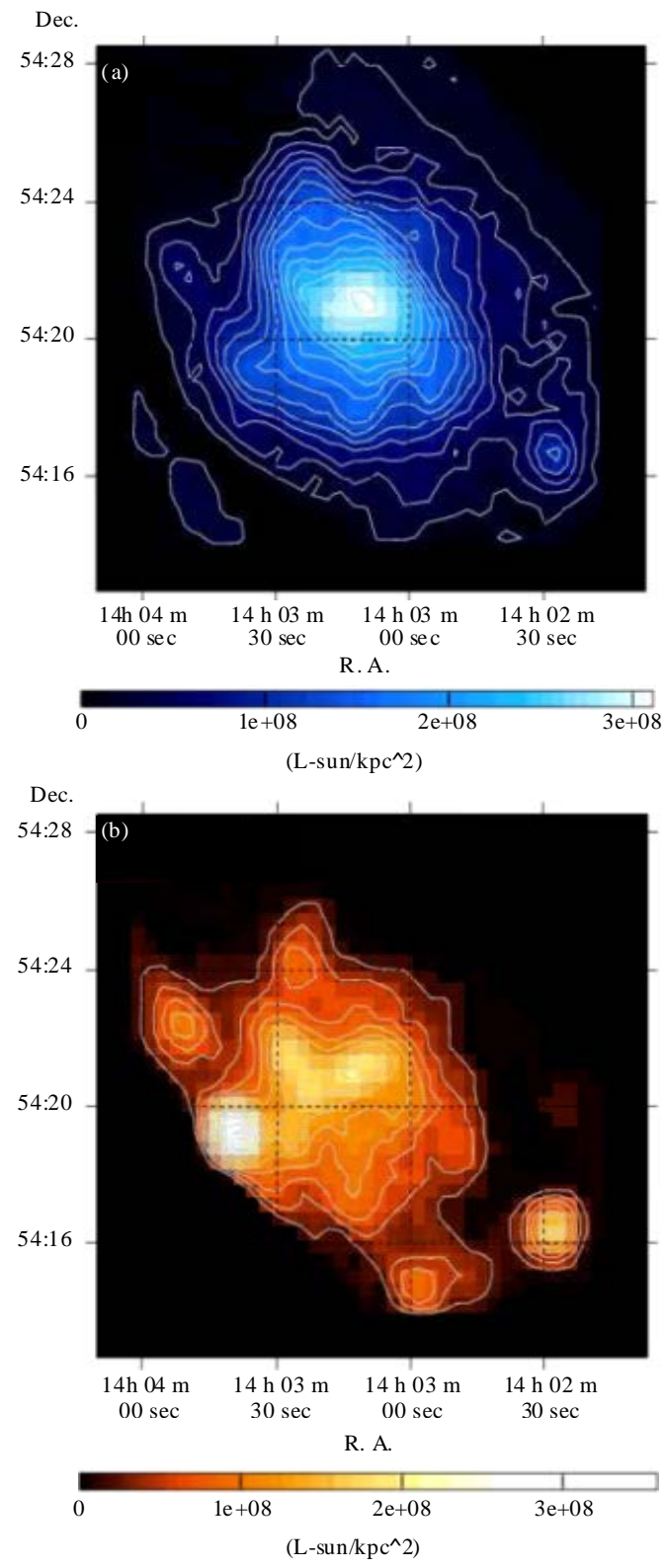

Fig. 3(a-b): Photographs of (a) Cold dust and (b) Hot dust recorded by AKARI-II 


\section{REFERENCES}

Doi, Y., M.E. Azkonaga, G. White, E. Figuered and Y. Chinone et al., 2009. AKARI far-infrared all sky survey. Proceedings of the Space Infrared Telescope for Cosmology and Astrophysics: Revealing the Origins of Planets and Galaxies Joint European Japanese Workshop, July 6-8, 2009, Oxford, UK., pp: 1-5.

Kawada, M., H. Baba, P.D. Barthel, D. Clements and M. Cohen et al., 2007. The Far-Infrared Surveyor (FIS) for AKARI. J. Astron. Soc. Jpn., 59: 389-400.

Matsuura, S., M. Shirahata, M. Kawada, T.T. Takeuchi and D. Burgarella et al., 2011. Detection of the cosmic far-infrared background in akari deep field south. J. Astrophys., 12: 737-742.

Okamura, N., S. Hasegawa, F. Usui, T. Hiroi, T. Ootsubo, T.G. Muller and S. Sugita, 2014. Spectroscopic observations of dark main-belt asteroids in the 2.5-3. $1 \mu \mathrm{m}$ range. Proceedings of the 45th Lunar and Planetary Science Conference, March 17-21, 2014, Woodlands, Texas, pp: 1375-1378.
Onaka, T., I. Sakon, H. Fujiwara, T. Shimonishi and H.G. Lee et al., 2010. Recent results from the infrared satellite AKARI. Proceedings of the Interstellar Matter and Star Formation: A MultiWavelength Perspective, Astronomical Society of India Conference Series Volume 1, October 5-7, 2009, TIFR National Balloon Facility, Hyderabad, India, pp: 127-134.

Ootsubo, T., H. Kawakita, S. Hamada, H. Kobayashi, M. Yamaguchi and F. Usui, 2011. Survey of $\mathrm{CO}_{2}$ in 18 comets with the Japanese infrared satellite AKARI. J. EPSC Abstracts, 6: 365-369.

Pollo, A., T.T. Takeuchi, A. Solarz, P. Rybka, T.L. Suzuki, A. Pepiak and S. Oyabu, 2014. Clustering of far-infrared galaxies in the AKARI all-sky survey North. J. Earth Planets Space, 65: 1109-1116.

Yamamura, I. and T. de Jong, 2000. Warm gas around AGB stars. Proceedings of the Conference on ISO Beyond the Peaks, (ESA SP-456, 2000), February 2-4, 2000, Villafranca del Castillo, Spain, pp: 1-6. 